This article has been accepted for publication in Monthly Notices of the Royal Astronomical Society. (C: 2020 The Authors. Published by Oxford University Press on behalf of the Royal Astronomical Society. All rights reserved. 


\title{
LOFAR detection of a low-power radio halo in the galaxy cluster Abell 990
}

\author{
D. N. Hoang ${ }^{\circledR},{ }^{1 \star}$ T. W. Shimwell, ${ }^{2,3 \star}$ E. Osinga, ${ }^{3}$ A. Bonafede, ${ }^{4}$ M. Brüggen ${ }^{\circledR},{ }^{1}$ A. Botteon ${ }^{\circledR},{ }^{3}$ \\ G. Brunetti, ${ }^{5}$ R. Cassano, ${ }^{5}$ V. Cuciti, ${ }^{1}$ A. Drabent,${ }^{6}$ C. Jones, ${ }^{7}$ H. J. A. Röttgering ${ }^{3}$ and R. J. van Weeren ${ }^{3}$ \\ ${ }^{1}$ Hamburger Sternwarte, University of Hamburg, Gojenbergsweg 112, D-21029 Hamburg, Germany \\ ${ }^{2}$ Netherlands Institute for Radio Astronomy (ASTRON), PO Box 2, NL-7990 AA Dwingeloo, the Netherlands \\ ${ }^{3}$ Leiden Observatory, Leiden University, PO Box 9513, NL-2300 RA Leiden, the Netherlands \\ ${ }^{4}$ Department of Physics and Astronomy, Bologna University, Via Zamboni 33, I-40126 Bologna, Italy \\ ${ }^{5}$ IRA - INAF, Via P. Gobetti 101, I-40129 Bologna, Italy \\ ${ }^{6}$ Thüringer Landessternwarte, Sternwarte 5, D-07778 Tautenburg, Germany \\ ${ }^{7}$ Harvard-Smithsonian Center for Astrophysics, 60 Garden Street, Cambridge, MA 02138, USA
}

Accepted 2020 November 13. Received 2020 October 15; in original form 2020 May 18

\begin{abstract}
Radio haloes are extended $(\sim \mathrm{Mpc})$, steep spectrum sources found in the central region of dynamically disturbed clusters of galaxies. Only a handful of radio haloes have been reported to reside in galaxy clusters with a mass $M_{500} \lesssim 5 \times 10^{14} \mathrm{M}_{\odot}$. In this paper, we present a LOw Frequency ARray (LOFAR) $144 \mathrm{MHz}$ detection of a radio halo in the galaxy cluster Abell 990 with a mass of $M_{500}=(4.9 \pm 0.3) \times 10^{14} \mathrm{M}_{\odot}$. The halo has a projected size of $\sim 700 \mathrm{kpc}$ and a flux density of $20.2 \pm 2.2 \mathrm{mJy}$ or a radio power of $1.2 \pm 0.1 \times 10^{24} \mathrm{~W} \mathrm{~Hz}^{-1}$ at the cluster redshift $(z=0.144)$ that makes it one of the two haloes with the lowest radio power detected to date. Our analysis of the emission from the cluster with Chandra archival data using dynamical indicators shows that the cluster is not undergoing a major merger but is a slightly disturbed system with a mean temperature of $5 \mathrm{keV}$. The low X-ray luminosity of $L_{\mathrm{X}}=(3.66 \pm 0.08) \times 10^{44} \mathrm{erg} \mathrm{s}^{-1}$ in the $0.1-2.4 \mathrm{keV}$ band implies that the cluster is one of the least luminous systems known to host a radio halo. Our detection of the radio halo in Abell 990 opens the possibility of detecting many more haloes in poorly explored less massive clusters with low-frequency telescopes such as LOFAR, Murchison Widefield Array (MWA, Phase II), and upgraded Giant Metrewave Radio Telescope (uGMRT).
\end{abstract}

Key words: radiation mechanisms: non-thermal-galaxies: clusters: individual: Abell 990-galaxies: clusters: intracluster medium - diffuse radiation-large-scale structure of Universe.

\section{INTRODUCTION}

Diffuse ( $\sim \mathrm{Mpc}$ ) synchrotron radio emission (i.e. haloes) in the centre of galaxy clusters indicates the presence of relativistic particles and large-scale magnetic fields in the intracluster medium (ICM). It is well known that the acceleration of particles to relativistic energy and the amplification of magnetic fields at these scales are associated with dynamical events in the host cluster of galaxies. However, the physical mechanisms governing the particle acceleration and the magnetic field amplification have not been fully understood (e.g. Brunetti \& Jones 2014; van Weeren et al. 2019).

Radio haloes are extended $(\sim \mathrm{Mpc})$, faint $\left(\sim \mu \mathrm{Jy} \operatorname{arcsec}^{-2}\right.$ at $1.4 \mathrm{GHz})$, steep spectrum ${ }^{1}(\alpha \lesssim-1)$ sources that pervade large regions of the cluster. They are apparently unpolarized down to a few per cent above $\sim 1 \mathrm{GHz}$. In turbulent reacceleration model (e.g. Brunetti et al. 2001; Petrosian 2001; Brunetti \& Lazarian 2007), radio haloes are generated by merger-induced turbulence that accelerates cosmic ray (CR) particles and/or amplifies the magnetic fields in the ICM. In addition, hadronic models that attempt to explain the generation of relativistic particles by the inelastic collisions between

\footnotetext{
^E-mail: hoang@hs.uni-hamburg.de (DNH); shimwell@astron.nl (TWS)

${ }^{1}$ The convention $S \propto \nu^{\alpha}$ is used in this paper.
}

relativistic protons and thermal ions in the ICM seems to play a lesser role in the formation of radio haloes (Ackermann et al. 2010, 2016; Brunetti \& Jones 2014; Brunetti, Zimmer \& Zandanel 2017), but could be a more significant process in minihaloes that have smaller sizes $(\lesssim 500$ kpc; Pfrommer \& Enßlin 2004; Brunetti \& Jones 2014; Zandanel, Pfrommer \& Prada 2014).

Observational evidence shows that the generation of radio haloes depends on the dynamical state of the host clusters and their mass (e.g. Wen \& Han 2013; Cuciti et al. 2015). Radio haloes are more likely to be observed in clusters that are massive and highly disturbed.

The power of radio haloes is known to roughly correlate with their cluster mass and X-ray luminosities (e.g. Cassano et al. 2013; Bîrzan et al. 2019). The scatters in the correlations reflect the evolution of haloes and dynamical state of clusters (e.g. Cuciti et al. 2015). Spectral properties of the haloes are also a contributing factor to the scatters. According to turbulent reacceleration models these properties reflect the efficiency of particle acceleration and are connected with cluster mass and dynamics. Steep spectrum haloes are generally expected in less massive systems and at higher redshift, and are thought to be less powerful sitting below the radio power-cluster mass correlation (e.g. Cassano, Brunetti \& Setti 2006; Brunetti et al. 2008; Cassano et al. 2010b, 2013).

Our understanding of the formation of radio haloes is mainly based on studies of galaxy clusters that are more massive than $5 \times 10^{14} \mathrm{M}_{\odot}$. 
Table 1. Observation details.

\begin{tabular}{lcc}
\hline Telescope & LOFAR $144 \mathrm{MHz}^{a}$ & VLA 1.5 GHz \\
\hline Project/pointing & LC4_034/P154+50, P156+47, P158+50 & $15 \mathrm{~A}-270$ \\
Configurations & HBA_DUAL_INNER & B \\
Observing dates & 2015 Aug 8, 2015 July 21, 2015 June 8 & Feb 22, 2015 \\
Obs IDs & L345594, L351840, L345592 & - \\
Calibrators & $3 \mathrm{C} 196$ & $3 \mathrm{C} 196,3 \mathrm{C} 286$ \\
Frequency (MHz) & $120-168$ & $1000-2000$ \\
Bandwidth (MHz) & 48 & 1000 \\
On-source time (h) & 24 & 0.6 \\
Integration time (s) & 1 & 3 \\
Frequency resolution (kHz) & 12.2 & 1000 \\
Correlations & $\mathrm{XX}, \mathrm{XY}, \mathrm{YX}, \mathrm{YY}$ & $\mathrm{RR}, \mathrm{RL}, \mathrm{LR}, \mathrm{LL}$ \\
Number of stations/antennas & $59,60,59$ & 26 \\
\hline
\end{tabular}

${ }^{a}$ For a detail description of the LoTSS data, see Shimwell et al. $(2017,2019)$.

There have been only a handful of radio haloes detected in clusters that are less massive than $5 \times 10^{14} \mathrm{M}_{\odot}$ (Abell 545, Giovannini, Tordi \& Feretti 1999; Bacchi et al. 2003; Abell 141, Duchesne et al. 2017; Abell 2061, Rudnick \& Lemmerman 2009; Abell 2811, Duchesne et al. 2017; Abell 3562, Venturi et al. 2000, 2003; Giacintucci et al. 2005; PSZ1 G018.75+23.57, Bernardi et al. 2016; RXC J1825.3+3026, Botteon et al. 2019; Abell 2146, HlavacekLarrondo et al. 2018; Hoang et al. 2019). This is due to the sensitivity limitation of previous radio observations that are unable to detect faint radio haloes in low-mass clusters. As a consequence, radio haloes in the regime of low-mass clusters are largely unexplored. Steep spectrum nature of radio haloes makes low-frequency telescopes such as LOFAR (van Haarlem et al. 2013) ideal instruments for studying radio haloes in the low-mass regime.

In this paper, we present our search for extended radio emission from the galaxy cluster Abell 990 (hereafter A990). With a mass of $M_{500}=(4.9 \pm 0.3) \times 10^{14} \mathrm{M}_{\odot}$ (Planck Collaboration XXVII 2016), A990 is an excellent target to search for cluster-scale radio emission in a mass range that is below the typical one where radio haloes are found. We make use of the LOFAR Two-metre Sky Survey (LoTSS; Shimwell et al. 2017, 2019) 120-168 MHz data. The Karl G. Jansky Very Large Array (VLA) 1-2 GHz data and the archival NRAO VLA Sky Survey (NVSS) $1.4 \mathrm{GHz}$ data are used to constraint the spectral properties the extended radio sources. In addition, we also use the archival X-ray Chandra data (Obs ID: 15114) to study dynamical state of the cluster.

We assume a flat $\Lambda$ cold dark matter $(\Lambda \mathrm{CDM})$ cosmology with $\Omega_{\mathrm{M}}=0.3, \Omega_{\Lambda}=0.7$, and $H_{0}=70 \mathrm{~km} \mathrm{~s}^{-1} \mathrm{Mpc}^{-1}$. With the adopted cosmology, 1 arcmin corresponds to a physical size of $151.6 \mathrm{kpc}$ at the cluster redshift, i.e. $z=0.144$. The luminosity distance to the cluster is $D_{L}=682.1 \mathrm{Mpc}$.

\section{OBSERVATIONS AND DATA REDUCTION}

\subsection{LOFAR data}

LoTSS is an on-going 120-168 MHz survey of the entire Northern hemisphere (Shimwell et al. 2017, 2019). As of 2020 March 4, 1522 of 3168 pointings have been observed. The calibrated data covering 27 per cent of the Northern sky will be released in the upcoming Data Release 2. A990 was observed with LOFAR, as part of LoTSS, during Cycle 4 and is covered by three pointings: P154+50, P156+47, and $\mathrm{P} 158+50$. Details of the observations are given in Table 1 .

The LOFAR data were calibrated in two steps to correct for the direction-independent and direction-dependent effects using
PREFACTOR $^{2}$ (van Weeren et al. 2016; Williams et al. 2016; de Gasperin et al. 2019) and DDF-PIPELINE ${ }^{3}$ (Tasse 2014a,b; Smirnov \& Tasse 2015; Tasse et al. 2018; Shimwell et al. 2019), respectively. We briefly outline the procedure below. For a full description of the procedure, we refer to Shimwell et al. (2019).

In the direction-independent calibration, the absolute amplitude is corrected according to the Scaife \& Heald (2012) flux scale. The calibration solutions are derived from the observations of 3C 196 that model has a total flux density of $83.1 \mathrm{Jy}$ at $150 \mathrm{MHz}$. The initial $\mathrm{XX}-\mathrm{YY}$ phase and clock offsets for each station are derived from the amplitude calibrator and are transferred to the target data. The target data are flagged and corrected for ionospheric Faraday rotation. ${ }^{4}$ During the data processing, the radio frequency interference (RFI) is removed with AOFLAGGER (Offringa, van de Gronde \& Roerdink 2012) when needed. The data are then phase calibrated against a wide-field sky model that is extracted from the GMRT $150 \mathrm{MHz}$ AllSky Radio Survey (TGSS-ADR1) catalogue (Intema et al. 2017).

The direction-dependent calibration step mainly aims to correct for the directional phase distortions caused by the ionosphere and the errors in the primary beam model of the LOFAR High Band Antenna (HBA). The direction-dependent corrections are solved for each antenna in $\mathrm{KMS}^{5}$ (Tasse 2014a,b; Smirnov \& Tasse 2015) implemented in the DDF-PIPELINE. The flux densities of the LOFARdetected sources are corrected with the bootstrapping technique using the VLA Low-Frequency Sky Survey Redux (VLSSr) and Westerbork Northern Sky Survey (WENSS) catalogues (Hardcastle et al. 2016). Prior to the bootstrapping, the WENSS catalogue is scaled by a factor of 0.9 to be consistent with the flux density scale in LoTSS (e.g. Scaife \& Heald 2012). To improve the image quality, the pipeline uses a 'lucky imaging' technique that generates additional weights to the visibilities basing on the quality of the calibration solutions (Bonnassieux et al. 2018). For imaging, DDFACET imager (Tasse et al. 2018) is used to deconvolve the calibrated data on each facet.

To improve the quality of the final images in the cluster region, the data are processed through 'extraction' and self-calibration steps. In the extraction, all sources outside of the cluster region are subtracted because they are not of the interest of this work. The sources outside of a $40 \times 40 \operatorname{arcmin}^{2}$ box centred at the cluster are subtracted using

\footnotetext{
${ }^{2}$ https://www.astron.nl/citt/prefactor

${ }^{3}$ https://github.com/mhardcastle/ddf-pipeline

${ }^{4}$ https://github.com/lofar-astron/RMextract

${ }^{5}$ https://github.com/saopicc/killMS
} 
Table 2. Imaging parameters.

\begin{tabular}{|c|c|c|c|c|}
\hline Data & $\begin{array}{c}u v \text { range } \\
(\mathrm{k} \lambda)\end{array}$ & $\begin{array}{c}\text { ROBUST }^{a} \\
\text { (UV-TAPER) }\end{array}$ & $\begin{array}{c}\theta_{\mathrm{FWHM}} \\
{\left[\operatorname{arcsec}^{2}(\mathrm{PA})^{b}\right]}\end{array}$ & $\begin{array}{c}\sigma \\
\left(\mu \mathrm{Jy} \mathrm{beam}^{-1}\right)\end{array}$ \\
\hline \multirow[t]{2}{*}{ LOFAR } & $0.15-65$ & $\begin{array}{c}-0.25 \\
(5 \operatorname{arcsec})\end{array}$ & $\begin{array}{c}16.6 \times 8.9 \\
\left(84^{\circ}\right)\end{array}$ & 80 \\
\hline & $0.15-65$ & $\begin{array}{c}0.25 \\
(10 \operatorname{arcsec})\end{array}$ & $\begin{array}{c}60.4 \times 46.7 \\
\quad\left(-73^{\circ}\right)\end{array}$ & 180 \\
\hline VLA & $0.52-74.6$ & $\begin{array}{c}0.25 \\
(10 \operatorname{arcsec})\end{array}$ & $\begin{array}{c}10.9 \times 8.6 \\
\left(68^{\circ}\right)\end{array}$ & 38 \\
\hline
\end{tabular}

${ }^{a}$ Briggs weighting of the visibility. ${ }^{b}$ Position angle.

the directional calibration solutions from the DDF-PIPELINE run. After the subtraction, data from all pointings are phase shifted to the cluster position, averaged in time and frequency, and compressed with Dysco (Offringa 2016) to reduce data size. The data are corrected for the primary beam attenuation in the direction of the cluster by multiplying with a factor that is inversely proportional to the station beam response. The requirement for the beam correction here is that the region of interest (i.e. the angular size of the cluster) is small enough for the station beam response to be approximately uniform. In addition, the total flux density in the extraction region should be above $\sim 0.3$ Jy for the calibration to be converged. Details of the 'extraction' and self-calibration steps are described in van Weeren et al. (2020).

The combined data sets are processed with multiple selfcalibration iterations including 'tecandphase' and gain calibration rounds. After each iteration, the calibration solutions are smoothed with LOSoTo $^{6}$ (de Gasperin et al. 2019) to minimize the effect of noisy solutions. The final intensity images of the cluster at different resolution are created using WSCLEAN ${ }^{7}$ (Offringa et al. 2014) with the imaging parameters in Table 2. To enhance extended sources, we subtract compact sources from the $u v$ data and make images of the cluster with more weightings on the short $u v$ baselines. The models for compact sources used in the subtraction are obtained from reimaging the cluster using only data with $u v$ distance longer than $1.0 \mathrm{k} \lambda$, which do not contain extended emission larger than 4.2 arcmin (i.e. $637 \mathrm{kpc}$ at the cluster redshift).

To examine the flux scale in the LOFAR images, we compare the flux densities of nearby compact sources with those in the TGSSADR1 $150 \mathrm{MHz}$ survey (Intema et al. 2017). The selected sources have a flux densities above $20 \sigma$ in both LOFAR and TGSS-ADR1 images. We found that the flux scale in our LOFAR image is about 4 per cent higher than the scale in the TGSS-ADR1 image. We adapt in our analysis a flux scale uncertainty of 10 per cent.

\subsection{VLA data}

The VLA L-band (1-2 GHz) data were taken on 2015 February 22 while the telescope was in the B-array configuration. The target was observed for four scans of approximately $10 \mathrm{~min}$ each, with a few hours in between the scans. The calibration is processed in the standard fashion (flux, phase, amplitude, band-pass, etc.) with CASA ${ }^{8}$ using 3C 147 and 3C 286 as calibrators. The flux density scale was set according to the Perley \& Butler (2017) flux scale. The RFI was automatically removed by the CASA 'TFCROP' and 'FLAGDATA' tasks. To eliminate the remaining RFI after the CASA flaggers, the
AOFLAGGER (Offringa et al. 2012) was employed. Additionally, outlier visibilities were looked for in the amplitude- $u v$ distance plane by inspecting the cross-hand (RL, LR) correlations. Since most radio sources are generally not polarized, the cross-hand correlations are a good additional indicator of RFI. In this plane, visibilities more than $6 \sigma$ away from the mean were flagged.

Finally, to remove residual amplitude and phase errors and increase the quality of the image, we performed self-calibration of the target field. Three rounds of phase calibration and three rounds of amplitude and phase calibration with the decrease of the solution interval each round were done. Amplitude solutions with a signal-to-noise ratio smaller than 3 were flagged, as well as outlier ( $>3 \sigma$ away from mean) amplitude solutions. The remaining RFI is manually flagged before the calibrated data is imaged with WSCLEAN (Offringa et al. 2014). The final images are corrected for the attenuation of the primary beam by dividing the image pixel values by the VLA primary beam model created by CASA.

\subsection{Chandra data}

Chandra observations of A990 were performed for $10 \mathrm{ks}$ on 2013 June 3 with the AXAF CCD Imaging Spectrometer (ObsID: 15114). The data were calibrated using the CLUSTERPYXT automatic pipeline $^{9}$ (Alden et al. 2019) that calls pre-built routines in CIAO ${ }^{10}(\mathrm{Fr}-$ uscione et al. 2006) and SHERPA ${ }^{11}$ (Freeman, Doe \& Siemiginowska 2001). The pipeline is able to generate $X$-ray surface brightness, spectral temperature, pressure, particle density, and entropy maps from Chandra observations with minimal inputs from users. The pipeline retrieves the data and corresponding backgrounds from the Chandra Data Archive. As our interest is X-ray extended emission from the cluster, point sources are manually removed from the data. Highenergy events are identified and filtered out by the pipeline. The gas temperature and pressure are obtained by spectral fitting of the events in the regions of interest. CLUSTERPYXT uses Astronomical Plasma Emission Code (APEC) model for optically thin collisionally ionized host plasma and a photoelectric absorption model (i.e. XSPEC PHABS) that includes redshift, metallicity, temperature, normalization, and hydrogen column density along the line of sight (Balucinska-Church \& McCammon 1992; Smith et al. 2001). We use a metallicity of $0.3 \mathrm{Z}_{\odot}$ (e.g. Werner et al. 2013) and a hydrogen column density of $1.31 \times 10^{20} \mathrm{~cm}^{-2}$ (Ben Bekhti et al. 2016) for the ICM. For details of the reduction procedure, we refer to Alden et al. (2019).

\section{RESULTS}

\subsection{Radio emission}

In Fig. 1, we present the LOFAR $144 \mathrm{MHz}$ radio emission contour maps of A990 at high and low resolution (see Table 2 for the image properties). The high-resolution contours show the detection of radio emission from galaxies that are labelled in Fig. 2. The radio emission from the brightest cluster galaxy (BCG; at $\alpha=10^{\mathrm{h}} 23^{\mathrm{m}} 39^{\mathrm{s}} .9, \delta=$ $+49^{\circ} 08^{\prime} 38^{\prime \prime} .4$ and redshift $z_{\mathrm{sp}}=0.14216 \pm 0.00005$; Alam et al. 2015 ) is also seen in the high-resolution image. Because of the low surface brightness of the cluster-scale emission, it is only seen in the low-resolution contours. In the subsections below, we present measurements for the detected sources.

\footnotetext{
${ }^{9}$ https://github.com/bcalden/ClusterPyXT

${ }^{10} \mathrm{https} / / / \mathrm{cxc}$.harvard.edu/ciao

${ }^{11}$ https://cxc.cfa.harvard.edu/sherpa/
} 


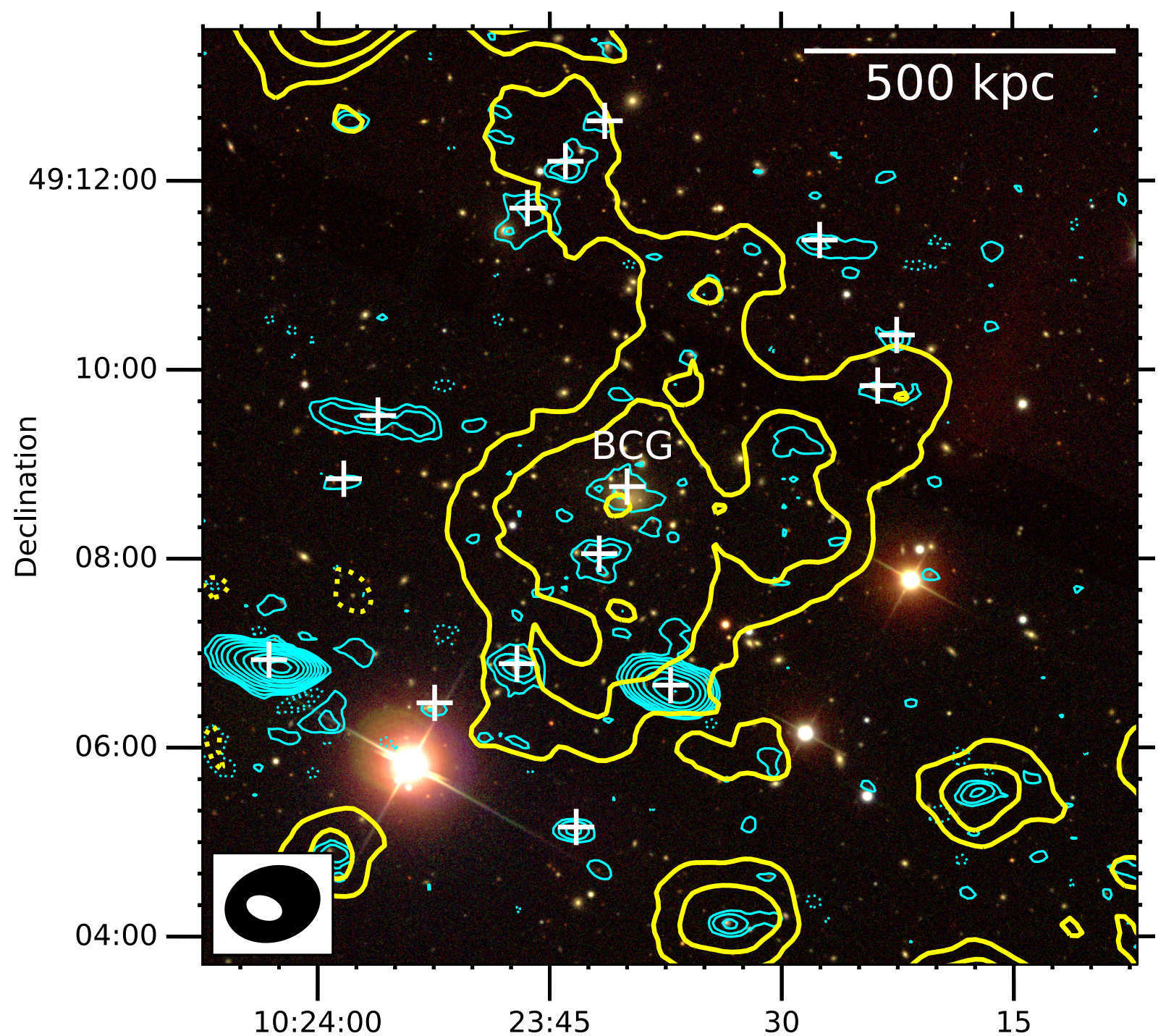

Right Ascension

Figure 1. LOFAR $144 \mathrm{MHz}$ high-resolution $\left(16.6 \times 8.9 \operatorname{arcsec}^{2}\right.$, cyan) and low-resolution $\left(60.4 \times 46.7 \operatorname{arcsec}^{2}\right.$, yellow) contours on the Sloan Digital Sky Survey (SDSS) optical ( $i, r$, and $g$ band) image. The contours are drawn at levels of $\pm[1,2,4,8,16,32] \times 3 \sigma$, where $\sigma=80$ and $_{180} \mu \mathrm{Jy}_{\text {beam }}{ }^{-1}$ for the highand low-resolution contours, respectively. Negative contours are shown by the dotted lines. Compact sources including the BCG marked with the plus $(+)$ are subtracted in the low-resolution contours. The synthesis beams are shown in the bottom left-hand corner. Source labels are given in Fig. 2.

\subsubsection{Extended radio source}

Fig. 1 shows that the extended radio emission is detected in the cluster centre, and its morphology is mainly extended in the northwest (NW)-south-east (SE) direction. The minimum and maximum projected sizes within the $3 \sigma$ contour are $515 \mathrm{kpc}$ and $960 \mathrm{Mpc}$, respectively. The projected size of the extended source is approximated as $D=\sqrt{515 \times 960 \mathrm{kpc}^{2}} \approx 700 \mathrm{kpc}$. The location and size of the extended source that mostly overlays the X-ray extended emission suggest that it is a radio halo.

The integrated flux density for the radio halo measured in the LOFAR low-resolution map is $S_{144 \mathrm{MHz}}=20.2 \pm 2.2 \mathrm{mJy}$. Here we select only pixels that are detected with $\geq 3 \sigma$ in the elliptical region in Fig. 2. We did not integrate the emission in the region of source $\mathrm{G}$ and $\mathrm{H}$ as this is likely the residuals of the imperfect subtraction of these sources. The error takes into account the image noise and the flux scale uncertainty of 10 per cent that are added in quadrature. The corresponding $144 \mathrm{MHz}$ power for the radio halo at $z=0.144$ is $P_{144 \mathrm{MHz}}=(1.2 \pm 0.1) \times 10^{24} \mathrm{~W} \mathrm{~Hz}^{-1}(k-$ corrected, assuming a spectral index of $\alpha=-1.3$, i.e. the mean indices for a number of known haloes; Feretti et al. 2012). To the best of our knowledge, A990 hosts one of the lowest radio power haloes at low $(\lesssim 300 \mathrm{MHz})$ frequencies known to date (together with RXC J1825.3+3026 studied by Botteon et al. 2019). If the halo has a steep spectrum, $\alpha \lesssim-1.3$, the radio power at $1.4 \mathrm{GHz}$ is $P_{1.4 \mathrm{GHz}} \lesssim(6.1 \pm 0.7) \times 10^{22} \mathrm{~W} \mathrm{~Hz}^{-1}$ that is still being one of the lowest powers for a radio halo at $1.4 \mathrm{GHz}$ (Bîrzan et al. 2019; Botteon et al. 2019). When all pixels above $2 \sigma$ are included, the values increase by about 12 per cent to $S_{144 \mathrm{MHz}}=22.7 \pm 2.4 \mathrm{mJy}$ and $P_{144 \mathrm{MHz}}=(1.3 \pm 0.1) \times 10^{24} \mathrm{~W} \mathrm{~Hz}^{-1}$. A similar difference in the flux density measurements within $2 \sigma$ and $3 \sigma$ contours was also seen in PSZ2 G099.86+58.45 (i.e. 15 per cent; Cassano et al. 2019). In this paper, we adopt the flux density measured with the $\geq 3 \sigma$ pixels for the radio halo in A990 (i.e. $S_{144 \mathrm{MHz}}=20.2 \pm 2.2 \mathrm{mJy}$ ). 


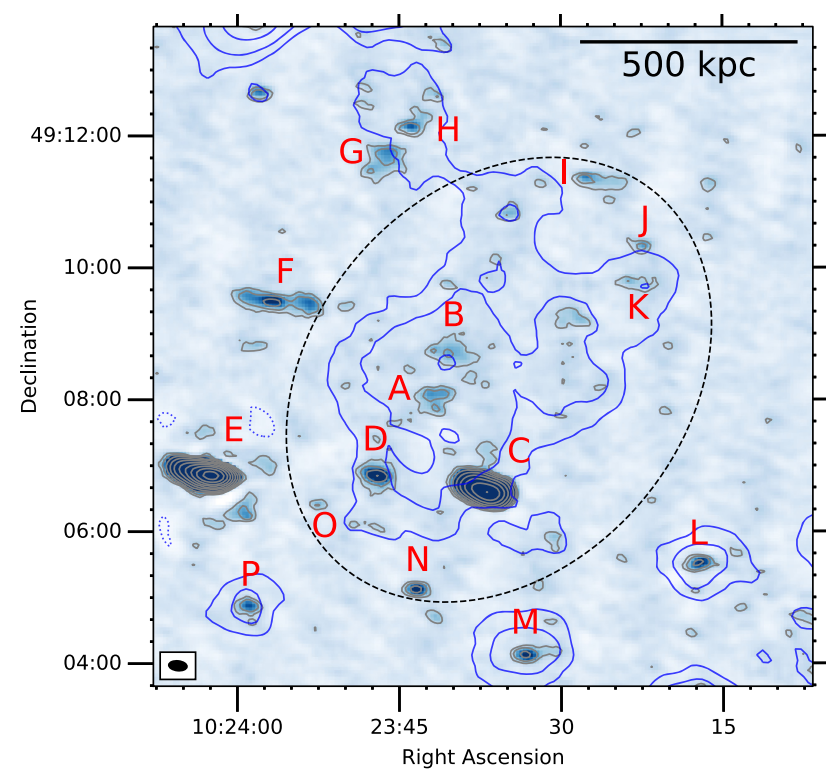

Figure 2. LOFAR $144 \mathrm{MHz}$ high-resolution image of A990. The highresolution (grey) and low-resolution (blue) contours are the same as those in Fig. 1. The ellipse is the region where the flux density of the halo is integrated. Compact sources are labelled.

The radio halo is undetected in the NVSS and VLA high-frequency images in Fig. 3. The non-detection could be due to the sensitivity of the NVSS and VLA data. To examine this possibility, we compute the expected surface brightness at 1.4 and $1.5 \mathrm{GHz}$, and compare it with the measurements in the NVSS and VLA images. Using the flux density measurement from the LOFAR data, we estimate the flux densities for the halo to be $S_{1.4 \mathrm{GHz}}=2.1 \mathrm{mJy}$ and $S_{1.5 \mathrm{GHz}}=2.0 \mathrm{mJy}$. Here we assume a flat spectrum, $\alpha=-1.0$, for the halo. This is a conservative approach as radio haloes are generally found to have steeper spectra $(\bar{\alpha} \approx-1.3$; Feretti et al. 2012). The true flux densities of the halo at $1.4 \mathrm{GHz}$ and $1.5 \mathrm{GHz}$ could be lower than these estimates (e.g. with $\alpha=-1.3, S_{1.4 \mathrm{GHz}}=1.1 \mathrm{mJy}$, and $S_{1.5 \mathrm{GHz}}=1.0 \mathrm{mJy}$ ). To calculate the expected surface brightness of the halo, we assume that the halo emission is uniformly distributed over the source area. Hence, the surface brightness is $I_{1.4 \mathrm{GHz}}=$ $S_{1.4 \mathrm{GHz}} / A=0.035 \mu \mathrm{Jy} \operatorname{arcsec}^{-2}$ and $I_{1.5 \mathrm{GHz}}=0.033 \mu \mathrm{Jy} \operatorname{arcsec}^{-2}$, where $A=61,075 \operatorname{arcsec}^{2}$ is the area of the halo (i.e. covered by the $\geq 3 \sigma$ in the LOFAR map). However, the sensitivity for the NVSS and VLA images is $\sigma=0.20 \mu \mathrm{Jy} \operatorname{arcsec}^{-2}$ (Condon et al. 1998) and $\sigma=0.36 \mu \mathrm{Jy} \operatorname{arcsec}^{-2}$ (see Table 2), respectively. These are not sensitive enough to detect the halo in A990. However, the radio emission at $144 \mathrm{MHz}$ is not constant over the halo (Fig. 3). The surface brightness emission at the peak region is $\sim 1.7$ times the mean pixel value in the halo region. In case that the emission at 1.4 and $1.5 \mathrm{GHz}$ follows the similar structure at $144 \mathrm{MHz}$, the surface brightness in some regions of the halo is expected to be higher, i.e. up to 0.06 and $0.05 \mu \mathrm{Jy} \operatorname{arcsec}^{-2}$, respectively. The expected surface brightness is still below the detection limit of the NVSS and VLA observations. We also note that the NVSS and VLA observations are not ideal for detecting faint, extended emission at the scales of the cluster. The NVSS data obtained from snapshot observations are poorly sampled in the $u v$ space. The VLA $1.5 \mathrm{GHz}$ observations in $\mathrm{B}$ configuration is also lacking of short baselines. To estimate the halo spectrum, future deep observations at high frequencies with the VLA (e.g. C, D configuration) and upgraded Giant Metrewave Radio
Telescope (uGMRT) or at low frequencies with LOFAR Low Band Antennas (10-80 MHz) will be necessary.

\subsubsection{Compact radio sources}

A number of compact radio sources are detected with our LOFAR observations in Fig. 1. In Table 3, we give the flux densities and spectra between $144 \mathrm{MHz}$ and $1.5 \mathrm{GHz}$ for the sources within a field of view of $\sim 10$ arcmin centred on the cluster. The spectral indices of the compact sources range from -0.50 to -1.84 . The redshifts of sources B, D, K, and P range from 0.1326 to 0.15073 implying that they are at similar redshifts of the cluster members $(\bar{z}=0.144)$. The overlaid radio-Sloan Digital Sky Survey (SDSS) optical images of the compact sources are shown in Fig. A1. Sources A, E, G, H, I, L, and $\mathrm{N}$ do not have clear SDSS optical counterparts. Sources A and B consist of multiple point sources. Sources $\mathrm{G}$ and $\mathrm{H}$ are likely two lobes of a Fanaroff-Riley type I (FR I) active galactic nucleus.

\subsection{X-ray emission}

The Chandra $0.5-2.0 \mathrm{keV}$ image of the cluster is shown in Fig. 4. The X-ray emission from the cluster is relatively concentrated in the central region. Its peak emission is closed to the location of the BCG. In the outskirts, the X-ray emission has a lower surface brightness and is elongated in the north-east (NE)-south-west (SW) direction, which is perpendicular to the major axis of the LOFAR $144 \mathrm{MHz}$ radio emission. In the NE peripheral region of the cluster, X-ray emission is found, but radio emission is not detected. Reversibly, extended radio emission is detected in the west side of the cluster without X-ray emission seen. The total X-ray luminosity between energy range 0.1 and $2.4 \mathrm{keV}$ within an elliptical region in Fig. 4 is $L_{\mathrm{X}}=(3.66 \pm 0.08) \times 10^{44} \mathrm{erg} \mathrm{s}^{-1}$, implying that $\mathrm{A} 990$ is one of the lowest $\mathrm{X}$-ray luminosity clusters ever found to host a radio halo (Bîrzan et al. 2019). In the energy range 0.5-2.0 eV, the Xray luminosity is $L_{\mathrm{X}}=(2.45 \pm 0.06) \times 10^{44} \mathrm{erg} \mathrm{s}^{-1}$. The elliptical region is chosen to roughly cover the $\mathrm{X}$-ray $3 \sigma$ contours (i.e. $\sigma=1.5 \times 10^{-9}$ counts $\mathrm{cm}^{-2} \mathrm{~s}^{-1}$ ). At the measured X-ray luminosity the cluster is about eight times underluminous in the radio power compared with the $L_{\mathrm{X}}-P_{1.4 \mathrm{GHz}}$ correlation (e.g. Cassano et al. 2013).

To study dynamical status of the cluster, we use the X-ray data to derive morphological indicators and to construct a cluster temperature map.

\subsubsection{Dynamical status}

Morphological properties of X-ray emission are known to correlate with dynamical state of the host clusters of galaxies (e.g. Cassano et al. 2010b; Bonafede et al. 2017). The X-ray emission from A990 in Fig. 4 is mostly concentrated in the central region and is slightly elongated in the NE-SW direction that seems to indicate a slightly disturbed cluster but without major merging activities. We estimate a set of parameters to classify the merging status. These include (i) the concentration of the X-ray emission $c$ (Santos et al. 2008), (ii) the centroid shifts between the X-ray emission peak and the centroid of the X-ray emission within aperture sizes (e.g. Poole et al. 2006), and (iii) the power ratios $P_{m} / P_{0}(m=3$; Buote $\&$ Tsai 1995). These are briefly described below.

i. Concentration of X-ray emission is defined as $c=$ $S_{\mathrm{X}}\left(r_{1}<100 \mathrm{kpc}\right) / S_{\mathrm{X}}\left(r_{2}<500 \mathrm{kpc}\right)$, where $S_{\mathrm{X}}\left(r_{i}\right)$ is the total X-ray surface brightness within radius $r_{i}$. 

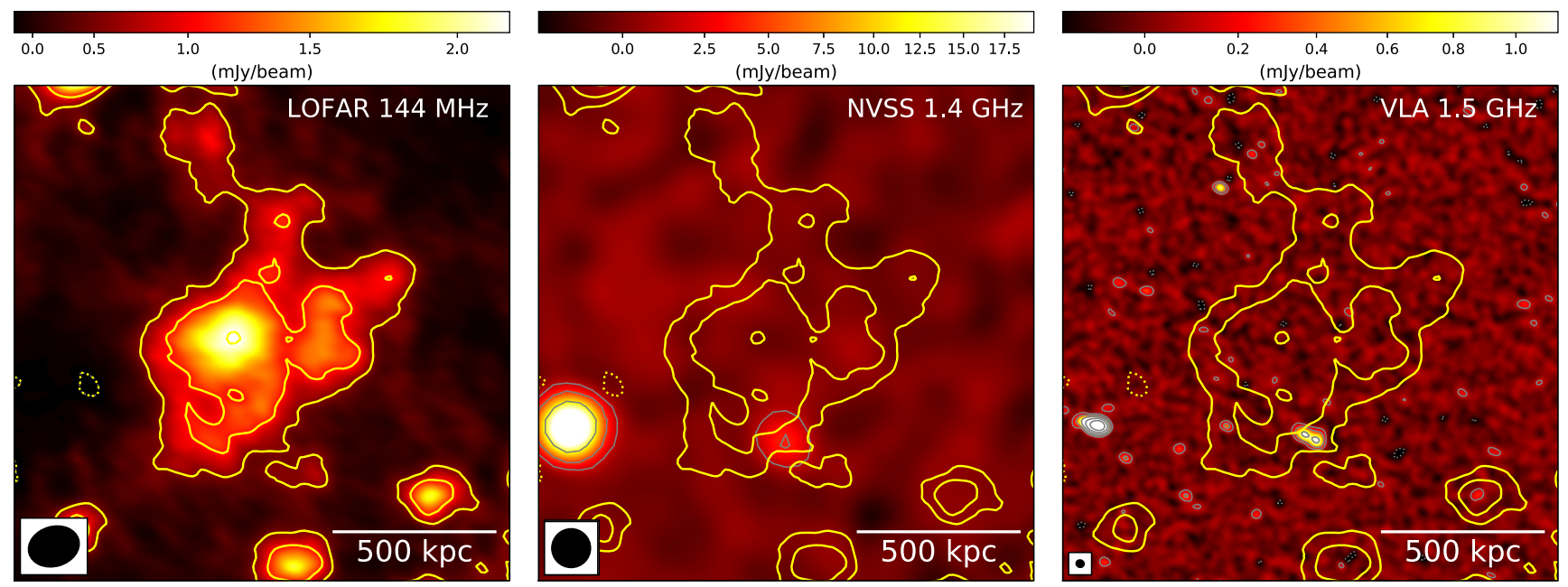

Figure 3. From left to right: LOFAR $144 \mathrm{MHz}$, NVSS $1.4 \mathrm{GHz}$, and VLA $1.5 \mathrm{GHz}$ image of A990. The NVSS and VLA (grey) contours are $\pm[1,3,9,27,81]$ $\times 3 \sigma$, where $\sigma=450$ and $38 \mu \mathrm{Jy}_{\text {beam }}^{-1}$ for the NVSS and VLA images, respectively. The field of view of the images and the LOFAR low-resolution (yellow) contours without compact sources are the same as those in Fig. 1.

Table 3. Flux densities and spectra for radio compact sources.

\begin{tabular}{|c|c|c|c|c|}
\hline Source & $S_{144 \mathrm{MHz}}(\mathrm{mJy})$ & $S_{1.5 \mathrm{GHz}}(\mathrm{mJy})$ & $\alpha$ & $z$ \\
\hline A & $1.77 \pm 0.19$ & $0.04 \pm 0.04$ & $-1.66 \pm 0.41$ & - \\
\hline B & $1.79 \pm 0.20$ & - & - & $0.14216 \pm 0.00005^{a}$ \\
\hline $\mathrm{C}$ & $75.10 \pm 7.51$ & $4.27 \pm 0.22$ & $-1.26 \pm 0.05$ & $0.6283 \pm 0.0356^{a}$ \\
\hline $\mathrm{D}$ & $3.07 \pm 0.32$ & $0.34 \pm 0.04$ & $-0.97 \pm 0.07$ & $0.1326 \pm 0.027^{a}$ \\
\hline E & $255.57 \pm 25.46$ & $34.74 \pm 1.74$ & $-0.88 \pm 0.05$ & - \\
\hline $\mathrm{F}$ & $3.08 \pm 0.32$ & $0.44 \pm 0.04$ & $-0.86 \pm 0.06$ & $0.5383 \pm 0.0303^{a}$ \\
\hline G & $2.33 \pm 0.25$ & $0.71 \pm 0.05$ & $-0.53 \pm 0.06$ & - \\
\hline $\mathrm{H}$ & $1.26 \pm 0.15$ & $0.18 \pm 0.04$ & $-0.87 \pm 0.11$ & - \\
\hline I & $1.31 \pm 0.15$ & $0.01 \pm 0.04$ & $-2.18 \pm 1.81$ & - \\
\hline $\mathrm{J}$ & $0.44 \pm 0.09$ & - & - & $0.6717 \pm 0.0655^{a}$ \\
\hline K & $0.59 \pm 0.10$ & - & - & $0.1326 \pm 0.0364^{a}$ \\
\hline $\mathrm{L}$ & $1.24 \pm 0.15$ & $0.24 \pm 0.04$ & $-0.73 \pm 0.09$ & - \\
\hline M & $1.52 \pm 0.17$ & $0.02 \pm 0.04$ & $-1.88 \pm 0.79$ & $0.1219 \pm 0.0206^{a}$ \\
\hline $\mathrm{N}$ & $1.08 \pm 0.13$ & $0.14 \pm 0.04$ & $-0.92 \pm 0.14$ & - \\
\hline $\mathrm{O}$ & $0.24 \pm 0.08$ & $0.21 \pm 0.04$ & $-0.06 \pm 0.17$ & $0.095 \pm 0.0481^{a}$ \\
\hline $\mathrm{P}$ & $0.93 \pm 0.12$ & - & - & $0.15073 \pm 0.00012^{b}$ \\
\hline
\end{tabular}

${ }^{a}$ Alam et al. (2015); ${ }^{b}$ Rines et al. (2013).

ii. Centroid shift $w=S D\left[\Delta_{i}\left(r_{i}\right)\right] / R_{\text {ap }}$, where $\Delta_{i}$ is the projected separation between the X-ray emission peak and the centroid of the $i$ th aperture of radius $r_{i}$.

iii. Power ratio $P_{m} / P_{0}$ presents the fraction of the $m$ th multipole moment to the total power gravitational potential. The lowest power ratio moment with $m=3$ gives a measure of the cluster substructures (e.g. Böhringer et al. 2010). For detailed formula, we refer to Buote \& Tsai (1995).

Several studies have used these morphological parameters to constrain the dynamical state of galaxy clusters (e.g. Böhringer et al. 2010; Cassano et al. 2010b; Bonafede et al. 2017; Savini et al. 2019). Clusters that are dynamically disturbed and host radio haloes have a low value of $c$ and high values of $w$ and $P_{3} / P_{0}$ (e.g. Cassano et al. 2010b; Cuciti et al. 2015), except some cases in Bonafede et al. (2014, 2015).

Following the calculations in Cassano et al. (2010b) and Bonafede et al. (2017), we estimate the morphological parameters for A990 to be $c=0.18, w=0.025$, and $P_{3} / P_{0}=2.17 \times 10^{-7}$. The X-ray emission peak used in the calculation is shown in Fig. 4. The centroid shift is calculated for circular apertures within $R_{\text {ap }}=500 \mathrm{kpc}$. The first aperture starts from $r_{i}=0.05 R_{\text {ap }}$ and the next ones increase in steps of $0.05 R_{\text {ap }}$. The power ratio $P_{3} / P_{0}$ is also calculated out to a radius of $R_{\text {ap }}$. We add our results to the morphological parameter diagrams in Fig. 5.

The morphological parameter diagrams in Fig. 5 show that A990 is located in the morphologically disturbed quadrant separated by the medians of $c, w$, and $P_{3} / P_{0}$ calculated from a sample of 32 galaxy clusters with X-ray luminosity $L_{X} \geq 5 \times 10^{44} \mathrm{erg} \mathrm{s}^{-1}$ and redshift $0.2 \leq z \leq 0.32$ (Cassano et al. 2010b). When adding more clusters from Bonafede et al. (2015) and from double relics from Bonafede et al. (2017), the medians are slightly shifted (see Fig. 5) and the points for A990 are closed to the boundaries for disturbed and relaxed clusters. This is in line with the idea that radio haloes are generated in disturbed galaxy clusters that supply energy via turbulence for the particle reacceleration and magnetic field amplification/generation. 


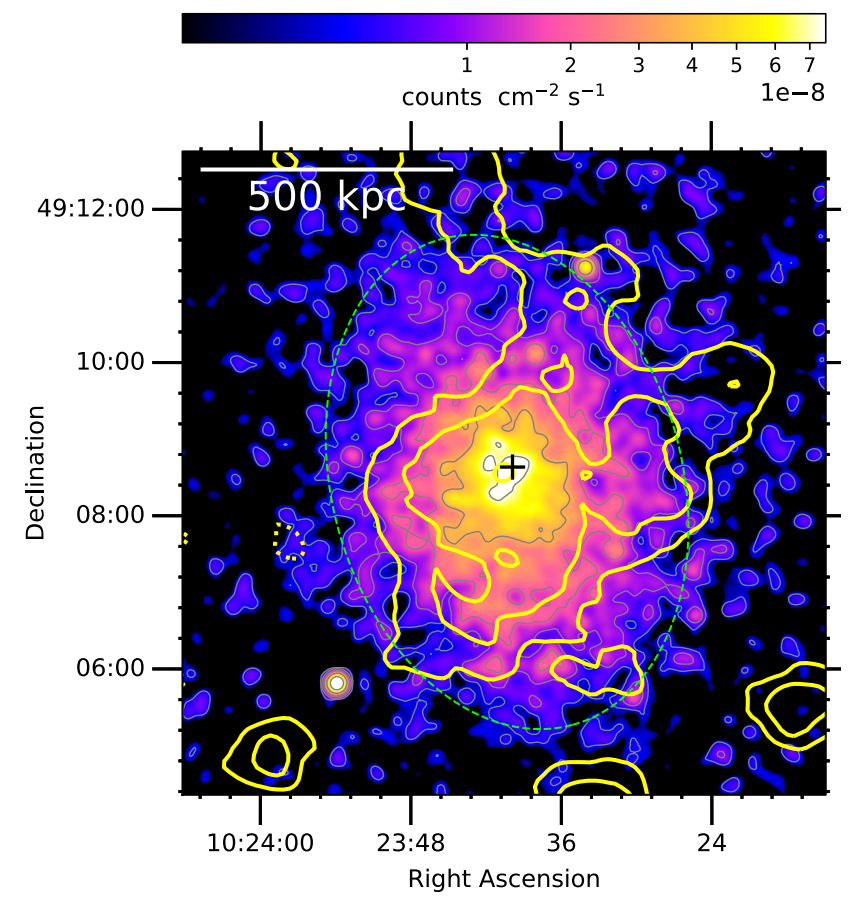

Figure 4. Chandra $0.5-2.0 \mathrm{keV}$ image $($ bin $=2)$ of the cluster overlaid with the LOFAR low-resolution contours. The Chandra data are smoothed with a Gaussian kernel of 5 pixels, where the pixel size is 0.98 arcsec. The $\mathrm{X}$ ray emission peaks at the plus $(+)$ location $\left(\mathrm{RA}=10^{\mathrm{h}} 23^{\mathrm{m}} 39^{\mathrm{s}} .9\right.$ and Dec. $=$ $+49^{\circ} 08^{\prime} 38^{\prime \prime}$. 4). The ellipse used for X-ray luminosity measurement is centred at $\mathrm{RA}=10^{\mathrm{h}} 23^{\mathrm{m}} 40^{\mathrm{s}} \cdot 4$, Dec. $=+49^{\circ} 08^{\prime} 25^{\prime \prime} .7$ with a position angle of $15^{\circ}$ The major and minor axes are 4.58 and 6.57 arcmin (i.e. 694 and $996 \mathrm{kpc}$ ), respectively.

\subsubsection{Temperature map}

We use CLUSTERPYXT package to perform spectral fitting of the Xray data and to produce temperature map in Fig. 6. The temperature map shows that the ICM gas has a mean temperature of about $5 \mathrm{keV}$ that is a moderate value for the known clusters (e.g. Frank et al. 2013; Zhu et al. 2016). Across the cluster, the distribution of the ICM gas temperature is patchy. The temperature is slightly higher in the NW region (i.e. $\sim 5.4 \mathrm{keV}$ ) than that in the $\mathrm{SE}$ region of the cluster (i.e. $\sim 5.1 \mathrm{keV}$ ). In the core region, the mean temperature is about $5.2 \mathrm{keV}$ around the X-ray emission peak and slightly increases to $5.4 \mathrm{keV}$ at the distance of $120 \mathrm{kpc}$. However, these variations are still within the mean errors of $\sim 0.55 \mathrm{keV}$ (see Fig. 6 , right). This is due to the short exposure duration of the X-ray observations (i.e. $10 \mathrm{ks}$ ). Future deep X-ray observations will provide information on the possible temperature variations in the cluster.

\section{DISCUSSION}

We have reported the detection of an extended radio source in A990 and classify it as a radio halo. The classification is based on (i) its extended size of $700 \mathrm{kpc}$ that is not associated with any compact sources, (ii) its location at the cluster centre, and (iii) its extended emission largely overlaid the X-ray extended emission from the ICM. A990 with a mass of $M_{500}=(4.9 \pm 0.3) \times 10^{14} \mathrm{M}_{\odot}($ Planck Collaboration XXVII 2016) is among the less massive clusters known to host a giant radio halo.

The power for radio haloes is well known to correlate with the mass and X-ray luminosity of their host clusters (e.g. Cassano et al.
2013). To examine the correlations in case of A990, we add our data points to the existing $P_{1.4 \mathrm{GHz}}-M_{500}$ and $P_{1.4 \mathrm{GHz}}-L_{500}$ correlations (see Fig. 7). Here $L_{500}$ is the X-ray luminosity measured within a radius $R_{500}$ at which the total particle density is 500 times the critical density of the Universe at the cluster redshift. If $\alpha=-1.0$, the radio power at $1.4 \mathrm{GHz}$ is $P_{1.4 \mathrm{GHz}}=1.2 \pm 0.1 \times 10^{23} \mathrm{~W} \mathrm{~Hz}^{-1}$ that is well below the expected value for a cluster that has a mass of $(4.9 \pm 0.3) \times 10^{14} \mathrm{M}_{\odot}$ (i.e. five times less power than expected) and X-ray luminosity of $(3.66 \pm 0.08) \times 10^{44} \mathrm{erg} \mathrm{s}^{-1}$ (i.e. four times underluminous in radio power). In case of $\alpha=-1.3$ (see Section 3.1), the halo power is lower, i.e. $P_{1.4 \mathrm{GHz}}=6.1 \times 10^{22} \mathrm{~W} \mathrm{~Hz}^{-1}$ and is significantly below the correlations (i.e. nine and eight times lower than expected from the $P_{1.4 \mathrm{GHz}}-M_{500}$ and $P_{1.4 \mathrm{GHz}}-L_{500}$ correlations, respectively) and the upper limit values for undetected haloes (Fig. 7). The difference could be that A990 lies in the poorly constrained regions for less massive clusters in the $P_{1.4 \mathrm{GHz}}-M_{500}$ and $P_{1.4 \mathrm{GHz}}$ $L_{500}$ correlations. However, it is still interesting that the halo in A990 is also about three times less powerful than the typical upper limits for haloes that are thought to have ultrasteep spectra (i.e. blue arrows in Fig. 7). Our morphological analysis of the cluster A990 with a mass of $(4.9 \pm 0.3) \times 10^{14} \mathrm{M}_{\odot}$ and its position in the $P_{1.4 \mathrm{GHz}}-M_{500}$ and $P_{1.4 \mathrm{GHz}}-L_{500}$ diagrams suggests that A990 can possibly host a radio halo with an ultrasteep spectrum. However, adequate higher or lower frequency observations are necessary to constrain its spectrum and confirm the scenario.

In the turbulence reacceleration model, radio haloes are formed through merger-induced turbulence and are likely to be detected in massive merging clusters. In these systems, a fraction of an amount of turbulent energy flux is channelled into the acceleration of relativistic particles and the amplification/generation of magnetic fields in the ICM during cluster mergers. In less massive clusters, merging events inject less energy into the ICM, resulting in radio haloes being formed less frequently and with steeper spectra (e.g. Brunetti et al. 2008; Cassano et al. 2010a, 2012). The model predicts that only about $10-15$ per cent of clusters with a mass below $6.5 \times 10^{14} \mathrm{M}_{\odot}$ host Mpc-scale radio haloes with spectra flatter than $\alpha \sim-1.5$ (e.g. Cuciti et al. 2015). At these masses, giant haloes with steeper spectra are expected to be more common and the fraction of these clusters with haloes increase up to 40 per cent including steeper spectrum haloes (e.g. Cassano et al. 2006, 2010a; Cuciti et al. 2015). Therefore, the detection of radio haloes in clusters with $M_{500} \lesssim$ $6.5 \times 10^{14} \mathrm{M}_{\odot}$ is expected to be more common at low frequencies with LOFAR (van Haarlem et al. 2013), Murchison Widefield Array (MWA, Phase II; Wayth et al. 2018), and uGMRT (Gupta et al. 2017). Our discovery of the radio halo in A990 with LOFAR and the fact that it sits below the correlations are in line with such expectations.

The low power of A990 in the $P_{1.4 \mathrm{GHz}}-M_{500}$ and $P_{1.4 \mathrm{GHz}}-L_{500}$ planes could also indicate a particular phase in the evolutionary state of the cluster. Magnetohydrodynamic (MHD) simulations of the reacceleration of cosmic ray electrons by merger-induced turbulence by Donnert et al. (2013) modelled the evolution of a radio halo power during cluster mergers. The radio halo becomes brighter after the merger and it is fainter in a later merging stage. At the same time the halo spectrum is flattened in the early stage of the merger, but is steepened below -1.5 after a few Gyr of core passage. The halo could be observed for a few Gyr depending on the observing frequencies that probe different energy of the population of relativistic electrons and magnetic field strength. At low frequencies, the halo is observable much longer due to the large lifetime of relativistic electrons with lower energy and weak magnetic fields. 

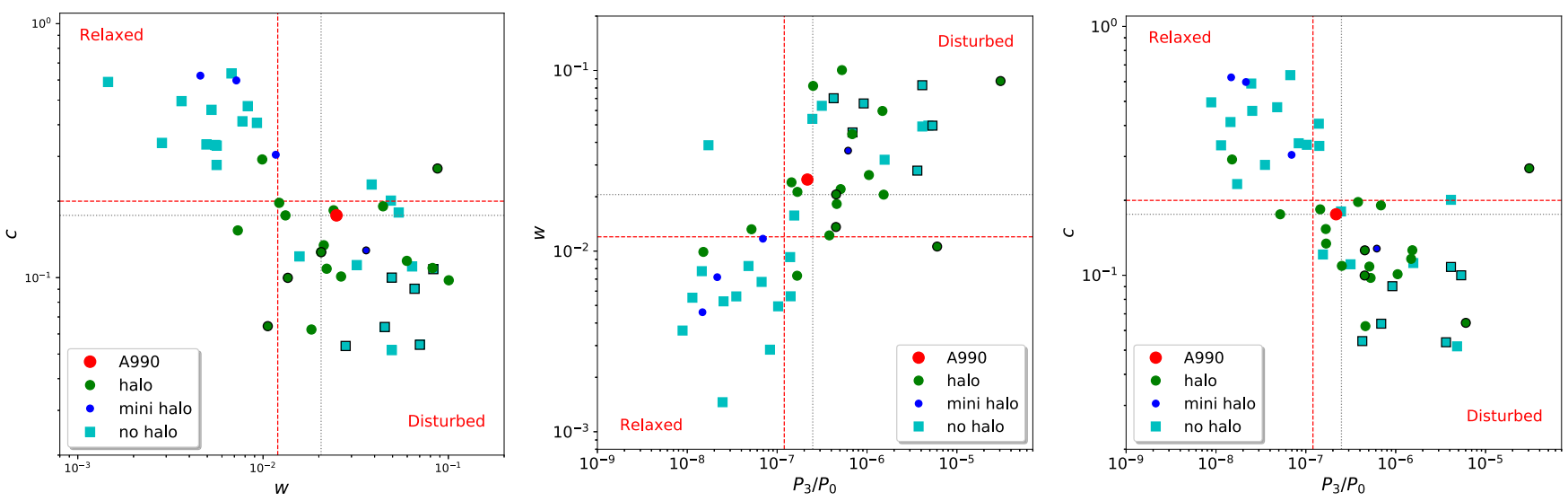

Figure 5. Morphological parameter diagrams for clusters with and without radio haloes (Cassano et al. 2010b; Bonafede et al. 2014, 2017). Clusters with radio haloes are shown with filled circles. Radio-quiet clusters are plotted with cyan squares. Clusters hosting double relics are additionally marked with black circles. The data points for A990 are marked with the red circles. The red dashed lines indicate the median values (i.e. $c=0.2, w=0.012, P_{3} / P_{0}=1.2 \times 10^{-7}$ ) calculated from a complete sample of 32 galaxy clusters (Cassano et al. 2010b). The black dotted lines (i.e. $c=0.18, w=0.021, P_{3} / P_{0}=2.5 \times 10^{-7}$ ) are calculated for all clusters in the plots.
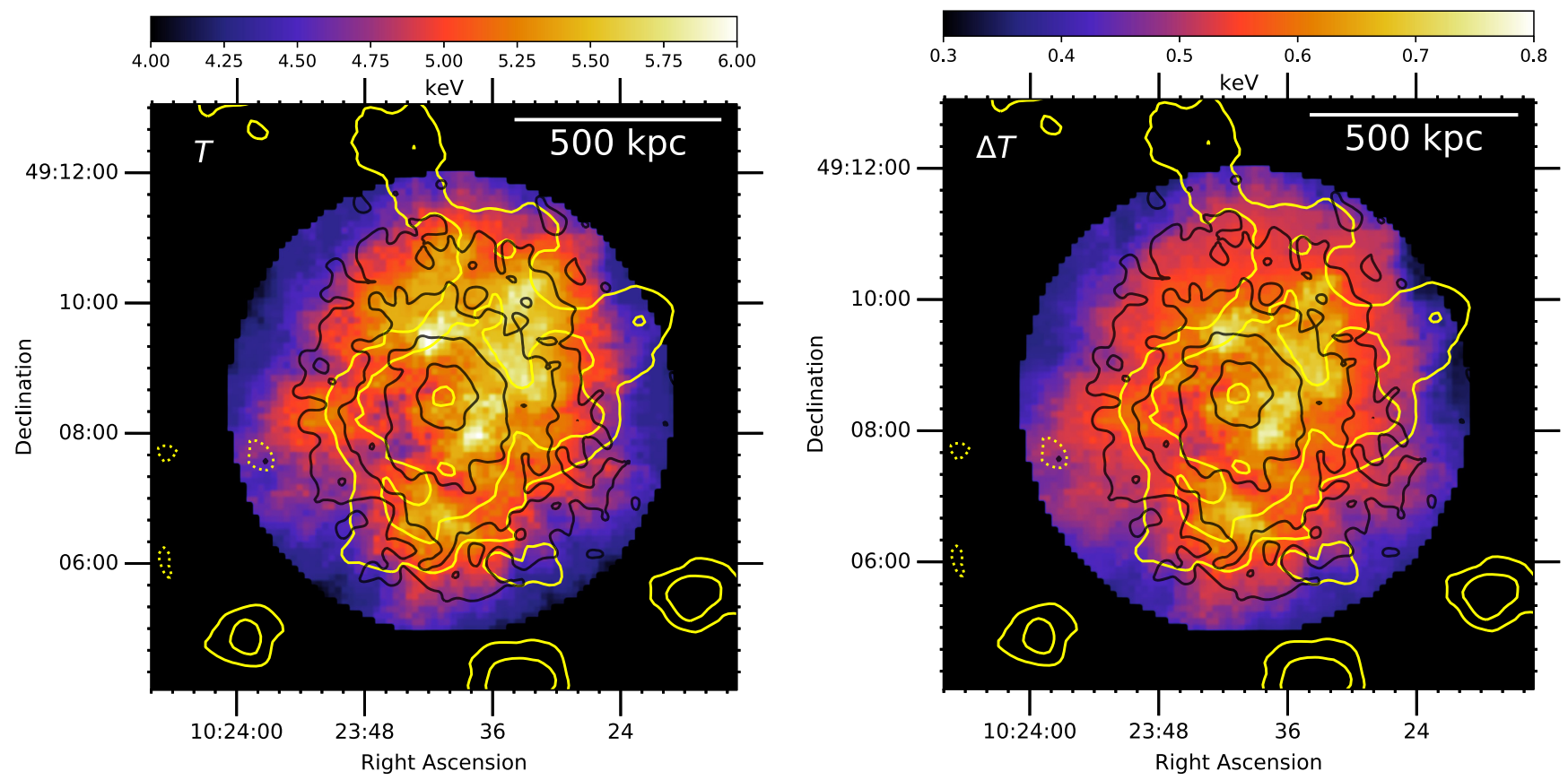

Figure 6. Temperature and error maps overlaid with the LOFAR low-resolution (yellow) and Chandra (black) contours. The contour levels are identical to those in Fig. 4. The cluster has a mean temperature of $5 \mathrm{keV}$.

In general, spectral information is thus crucial to understand the origin and evolution of radio haloes. On-going radio surveys of a large fraction of the sky such as LoTSS (Shimwell et al. 2017, 2019) will provide statistical estimates on the fraction of radio haloes in complete samples of clusters down to relatively small masses. In combination with high-frequency observations with the Australian Square Kilometre Array Pathfinder (ASKAP)/Evolutionary Map of the Universe (EMU; e.g. clusters with declination between $0^{\circ}$ and $+30^{\circ}$ ), uGMRT and VLA telescopes, the halo spectra will be measured that will improve our understanding of the mechanism of particle acceleration and magnetic field amplification/generation at cluster scales.

\section{CONCLUSIONS}

In this paper, we present LOFAR $144 \mathrm{MHz}$ observations, as part of the LoTSS surveys, of the galaxy cluster A990 $(z=0.144)$ with a mass of $M_{500}=(4.9 \pm 0.3) \times 10^{14} \mathrm{M}_{\odot}$. We detect an extended radio emission with a projected size of $\sim 700 \mathrm{kpc}$ at the cluster centre. Because of its location and size, we classify the extended radio emission as a radio halo.

The flux density at $144 \mathrm{MHz}$ for the halo is $S_{144 \mathrm{MHz}}=20.2 \pm$ $2.2 \mathrm{mJy}$ (i.e. above $3 \sigma$ contours), corresponding to a radio power of $P_{144 \mathrm{MHz}}=(1.2 \pm 0.1) \times 10^{24} \mathrm{~W} \mathrm{~Hz}^{-1}$ ( $k$-corrected). The radio halo is one of the two lowest power radio haloes detected to date. 

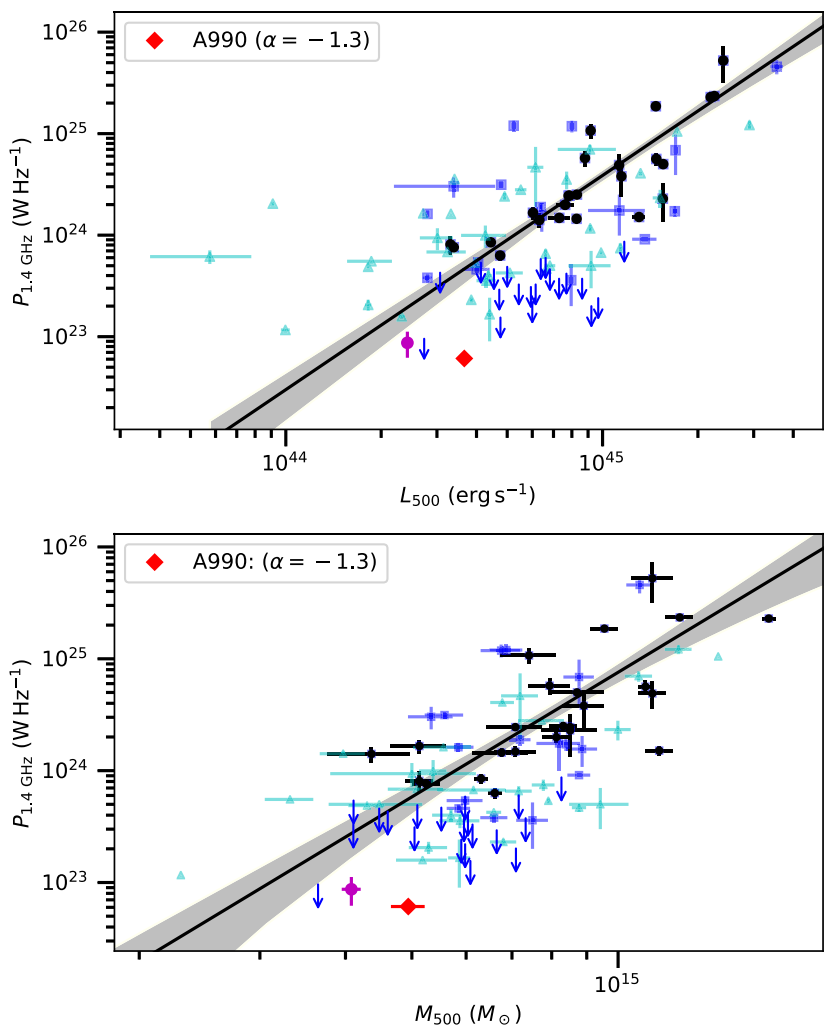

Figure 7. The $P_{1.4 \mathrm{GHz}}-M_{500}$ (bottom) and $P_{1.4 \mathrm{GHz}}-L_{500}$ (top) relations, adapted from Bîrzan et al. (2019). The data points for A990 and RXC J1825.3+3026 are shown by the red diamond and magenta circle, respectively. In both panels, the black circles, blue squares, and cyan triangles show radio haloes in the samples of Cassano et al. (2013), Martinez Aviles et al. (2016), and Bîrzan et al. (2019), respectively. The blue arrows indicate the power upper limits for undetected haloes in the Cassano et al. (2013), Kale et al. (2015), and Bîrzan et al. (2019) cluster samples. The solid lines are the best fits for the Cassano et al. (2013) haloes. The shadowed regions show the 95 per cent confidence region.

Our analysis on the dynamical status of the cluster using the $\mathrm{X}$ ray data reveals that the cluster has a slightly disturbed ICM and does not have major merger activities. The X-ray luminosity of the halo between 0.1 and $2.4 \mathrm{keV}$ is $L_{500}=(3.66 \pm 0.08) \times 10^{44} \mathrm{erg} \mathrm{s}^{-1}$. The cluster mean temperature is relatively low, at $5 \mathrm{keV}$. There are possible variations in the temperature, but require deep X-ray observations to confirm.

Our measurements on the radio power, X-ray luminosity, and the ICM temperature are at the low ends. This can be explained by the cluster being a dynamically disturbed, less massive system that contains less energy than more massive clusters.

Our study reveals the potential of detecting many faint radio haloes in less massive galaxy clusters with LOFAR. With the full coverage of the Northern hemisphere, the LoTSS survey will provide the fraction of clusters of this mass hosting radio haloes that will be compared with the theoretical predictions (e.g. Cassano et al. 2010a). A combination with high-frequency observations will provide spectral information on the sources that are important for studies of the origin of radio haloes.

\section{ACKNOWLEDGEMENTS}

We thank an anonymous referee for the helpful comments. NDH and $\mathrm{ABon}$ acknowledge support from the ERC through the grant
ERC-Stg DRANOEL 714245. MB acknowledges support from the Deutsche Forschungsgemeinschaft under Germany's Excellence Strategy - EXC 2121 'Quantum Universe' - 390833306. ABot, $\mathrm{EO}$, and RJvW acknowledge support from the VIDI research programme with project number 639.042.729, which is financed by the Netherlands Organisation for Scientific Research (NWO). HJAR acknowledges support from the ERC Advanced Investigator programme NewClusters 321271. AD acknowledges support by the BMBF Verbundforschung under the grant 05A17STA: The Jülich LOFAR Long Term Archive and the German LOFAR network are both coordinated and operated by the Jülich Supercomputing Centre (JSC), and computing resources on the supercomputer JUWELS at JSC were provided by the Gauss Centre for supercomputing e.V. (grant CHTB00) through the John von Neumann Institute for Computing (NIC). These data were (partly) processed by the LOFAR Two-Metre Sky Survey (LoTSS) team. This team made use of the LOFAR direction-independent calibration pipeline (https://github.com/lofar-astron/prefactor) that was deployed by the LOFAR e-infragroup on the Dutch National Grid infrastructure with support of the SURF Cooperative through grants e-infra 160022 and e-infra 160152 (Mechev et al. 2017). The LoTSS direction-dependent calibration and imaging pipeline (http://github.com/mhardcastle/ddf -pipeline) was run on compute clusters at Leiden Observatory and the University of Hertfordshire that are supported by a European Research Council Advanced Grant (NEWCLUSTERS-321271) and the UK Science and Technology Facilities Council (ST/P000096/1). We have including the Lotss-dr2-infrastructure list as this project made use of the DR2 pipeline and post-processing extraction/selfcal scheme. The scientific results reported in this paper are based on data obtained from the Chandra Data Archive. This research has made use of software provided by the Chandra X-ray Center (CXC) in the application packages CIAO, CHIPS, and SHERPA. The National Radio Astronomy Observatory is a facility of the National Science Foundation operated under cooperative agreement by Associated Universities, Inc.

\section{DATA AVAILABILITY}

The data underlying this paper will be shared on reasonable request to the corresponding author.

\section{REFERENCES}

Ackermann M. et al., 2010, ApJ, 717, L71

Ackermann M. et al., 2016, ApJ, 819, 149

Alam S. et al., 2015, ApJS, 219, 12

Alden B., Hallman E. J., Rapetti D., Burns J. O., Datta A., 2019, Astron. Comput., 27, 147

Bacchi M., Feretti L., Giovannini G., Govoni F., 2003, A\&A, 400, 465

Balucinska-Church M., McCammon D., 1992, ApJ, 400, 699

Ben Bekhti N. et al., 2016, A\&A, 594, A116

Bernardi G. et al., 2016, MNRAS, 456, 1259

Bîrzan L. et al., 2019, MNRAS, 487, 4775

Böhringer H. et al., 2010, A\&A, 514, A32

Bonafede A. et al., 2014, MNRAS, 444, L44

Bonafede A. et al., 2015, MNRAS, 454, 3391

Bonafede A. et al., 2017, MNRAS, 470, 3465

Bonnassieux E., Tasse C., Smirnov O., Zarka P., 2018, A\&A, 615, A66

Botteon A. et al., 2019, A\&A, 630, A77

Brunetti G., Jones T. W., 2014, Int. J. Modern Phys. D, 23, 1430007

Brunetti G., Lazarian A., 2007, MNRAS, 378, 245

Brunetti G., Setti G., Feretti L., Giovannini G., 2001, MNRAS, 320, 365

Brunetti G. et al., 2008, Nature, 455, 944 
Brunetti G., Zimmer S., Zandanel F., 2017, MNRAS, 472, 1506

Buote D. A., Tsai J. C., 1995, ApJ, 452, 522

Cassano R., Brunetti G., Setti G., 2006, MNRAS, 369, 1577

Cassano R., Brunetti G., Rottgering H. J. A., Bruggen M., 2010a, A\&A, 509, A68

Cassano R., Ettori S., Giacintucci S., Brunetti G., Markevitch M., Venturi T., Gitti M., 2010b, ApJ, 721, L82

Cassano R., Brunetti G., Norris R., Röttgering H., Johnston-Hollitt M., Trasatti M., 2012, A\&A, 548, A100

Cassano R. et al., 2013, ApJ, 777, 141

Cassano R. et al., 2019, ApJ, 881, L18

Condon J. J., Cotton W. D., Greisen E. W., Yin Q. F., Perley R. A., Taylor G. B., Broderick J. J., 1998, AJ, 115, 1693

Cuciti V., Cassano R., Brunetti G., Dallacasa D., Kale R., Ettori S., Venturi T., 2015, A\&A, 580, A97

de Gasperin F. et al., 2019, A\&A, 622, A5

Donnert J., Dolag K., Brunetti G., Cassano R., 2013, MNRAS, 429, 3564

Duchesne S. W., Johnston-Hollitt M., Offringa A. R., Pratt G. W., Zheng Q., Dehghan S., 2017, preprint (arXiv:1707.03517)

Feretti L., Giovannini G., Govoni F., Murgia M., 2012, A\&AR, 20, 54

Frank K. A., Peterson J. R., Andersson K., Fabian A. C., Sanders J. S., 2013, ApJ, 764, 46

Freeman P., Doe S., Siemiginowska A., 2001, Proc. SPIE, 4477, 76

Fruscione A. et al., 2006, Proc. SPIE, 6270, 62701V

Giacintucci S. et al., 2005, A\&A, 440, 867

Giovannini G., Tordi M., Feretti L., 1999, New Astron., 4, 141

Gupta Y. et al., 2017, Curr. Sci., 113, 707

Hardcastle M. J. et al., 2016, MNRAS, 462, 1910

Hlavacek-Larrondo J. et al., 2018, MNRAS, 475, 2743

Hoang D. N. et al., 2019, A\&A, 622, A21

Intema H. T., Jagannathan P., Mooley K. P., Frail D. A., 2017, A\&A, 598, A78

Kale R., Venturi T., Cassano R., Giacintucci S., Bardelli S., Dallacasa D., Zucca E., 2015, A\&A, 581, A23

Martinez Aviles G. et al., 2016, A\&A, 595, A116

Mechev A., Oonk J. B. R., Danezi A., Shimwell T. W., Schrijvers C., Intema H., Plaat A., Rottgering H. J. A., 2017, PoS, ISGC2017, 002

Offringa A. R., 2016, A\&A, 595, A99

Offringa A. R., van de Gronde J. J., Roerdink J. B. T. M., 2012, A\&A, 539, A95

Offringa A. R. et al., 2014, MNRAS, 444, 606

Perley R. A., Butler B. J., 2017, ApJS, 230, 7
Petrosian V., 2001, ApJ, 557, 560

Pfrommer C., Enßlin T. A., 2004, MNRAS, 352, 76

Planck Collaboration XXVII, 2016, A\&A, 594, A27

Poole G. B., Fardal M. A., Babul A., McCarthy I. G., Quinn T., Wadsley J., 2006, MNRAS, 373, 881

Rines K., Geller M. J., Diaferio A., Kurtz M. J., 2013, ApJ, 767, 15

Rudnick L., Lemmerman J. A., 2009, ApJ, 697, 1341

Santos J. S., Rosati P., Tozzi P., Böhringer H., Ettori S., Bignamini A., 2008, A\&A, 483, 35

Savini F. et al., 2019, A\&A, 622, A24

Scaife A. M. M., Heald G. H., 2012, MNRAS, 423, L30

Shimwell T. W. et al., 2017, A\&A, 598, A104

Shimwell T. W. et al., 2019, A\&A, 622, A1

Smirnov O. M., Tasse C., 2015, MNRAS, 449, 2668

Smith R. K., Brickhouse N. S., Liedahl D. A., Raymond J. C., 2001, ApJ, 556, L91

Tasse C., 2014a, preprint (arXiv:1410.8706)

Tasse C., 2014b, A\&A, 566, A127

Tasse C. et al., 2018, A\&A, 611, A87

van Haarlem M. P. et al., 2013, A\&A, 556, A2

van Weeren R. J. et al., 2016, ApJS, 223, 2

van Weeren R. J., de Gasperin F., Akamatsu H., Brüggen M., Feretti L., Kang H., Stroe A., Zandanel F., 2019, Space Sci. Rev., 215, 16

van Weeren R. J. et al., 2020, preprint (arXiv:2011.02387)

Venturi T., Bardelli S., Morganti R., Hunstead R. W., 2000, MNRAS, 314, 594

Venturi T., Bardelli S., Dallacasa D., Brunetti G., Giacintucci S., Hunstead R. W., Morganti R., 2003, A\&A, 402, 913

Wayth R. B. et al., 2018, Publ. Astron. Soc. Aust., 35, e033

Wen Z. L., Han J. L., 2013, MNRAS, 436, 275

Werner N., Urban O., Simionescu A., Allen S. W., 2013, Nature, 502, 656

Williams W. L. et al., 2016, MNRAS, 460, 2385

Zandanel F., Pfrommer C., Prada F., 2014, MNRAS, 438, 124

Zhu Z. et al., 2016, ApJ, 816, 54

\section{APPENDIX A: OPTICAL COUNTERPARTS OF COMPACT SOURCES}

To identify optical counterparts of the radio compact sources, we overlaid the LOFAR high-resolution contours on SDSS images in Fig. A1. 

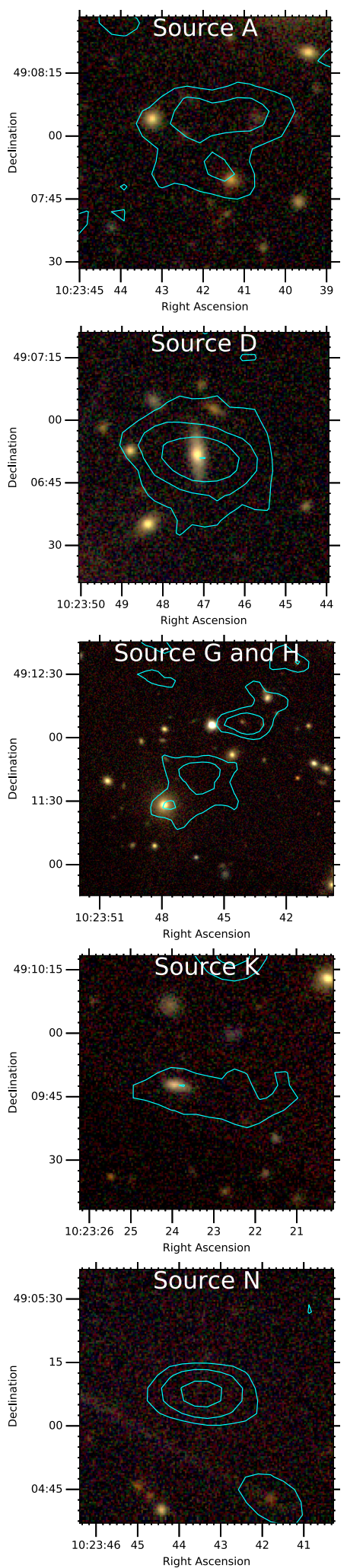
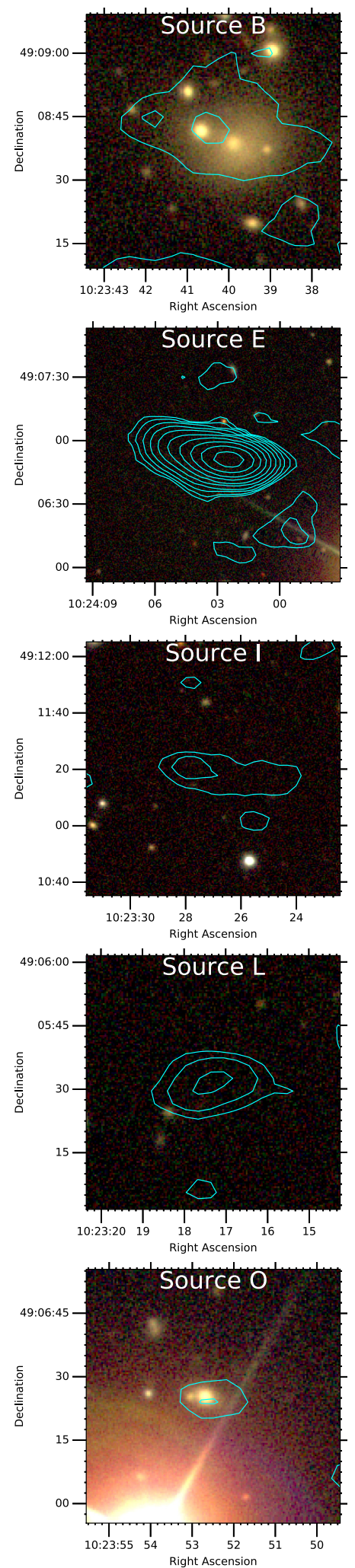
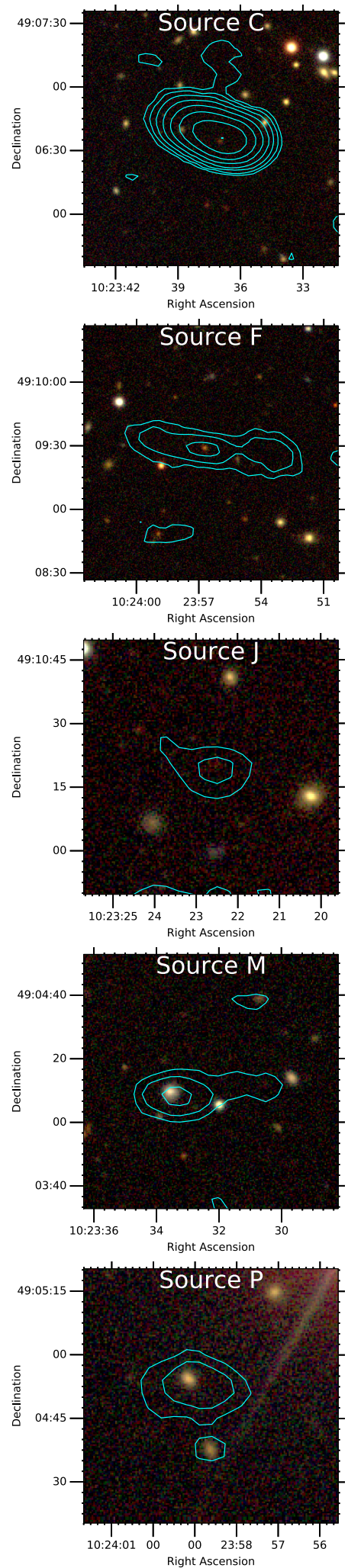

Figure A1. SDSS optical ( $i, r$, and $g$ band) images overlaid with the LOFAR high-resolution contours. The contours are at the same levels as those in Fig. 1 . The source labels are given in Fig. 2.

This paper has been typeset from a $\mathrm{T}_{\mathrm{E}} \mathrm{X} / \mathrm{LT} \mathrm{E} \mathrm{X}$ file prepared by the author. 